\title{
SEARCH FOR X RAYS FROM THE REGION OF THE ARIES-PERSEUS FLASHER
}

\author{
WALTER H. G. LEWIN \\ Physics Department, Massachusetts Institute of Technology, Cambridge, Massachusetts 02139 \\ JAN VAN PARAdiss ANd Eugene Damen \\ Astronomical Institute “Anton Pannekoek," University of Amsterdam, Roetersstraat 15, 1018WB, Amsterdam, The Netherlands
}

FRED JANSEN

Laboratory for Space Research Leiden, Postbus 9504, 2300 RA Leiden, The Netherlands

MARSHALl L. MCCALL

David Dunlap Observatory and University of Toronto, Box 360, Richmond Hill, Ontario L4C 4Y6, Canada

P. A. Feldman And K. F. TAPPing

Herzberg Institute of Astrophysics, National Research Council of Canada, 100 Sussex Drive, Ottawa K1A 0R6, Canada

Received 16 March 1987; revised 10 April 1987

\begin{abstract}
The region containing the Perseus flasher (Katz et al. 1986) was observed for $5.4 \mathrm{hr}$ with the EXOSAT observatory. Upper limits ( $2 \sigma$ levels of confidence) to a point source with a steady $\mathrm{x}$-ray flux were $2 \times 10^{-12} \mathrm{erg} \mathrm{cm}^{-2} \mathrm{~s}^{-1}(0.1-2 \mathrm{keV})$ and $6 \times 10^{-12} \mathrm{erg} \mathrm{cm}^{-2} \mathrm{~s}^{-1}(1-20 \mathrm{keV})$. Upper limits (4 $\sigma$ level of confidence) to $\mathrm{x}$-ray flashes of $\sim 1 \mathrm{~s}$ duration were $4 \times 10^{-9} \mathrm{erg} \mathrm{cm}^{-2} \mathrm{~s}^{-1}(0.1-2 \mathrm{keV})$ and $7 \times 10^{-10}$ erg $\mathrm{cm}^{-2} \mathrm{~s}^{-1}(1-20 \mathrm{keV})$.
\end{abstract}

\section{INTRODUCTION}

Between 1984 July and 1985 July, 24 bright optical flashes were detected visually near the border of the constellations Aries and Perseus by eight different observers at a total of 12 sites across Canada (Katz et al. 1986). One flash was photographed, and another was seen by two observers at different locations. The duration of the flashes was usually less than 1 s. The estimated positions of 20 of the events, and another seen in 1983, were close enough in the sky to suggest a common celestial origin. The photographed event, which was the brightest seen visually, reached $m_{v} \approx-1$ and lasted about 0.25 s. It was located in Perseus at $\alpha(1950)$ $=03^{\mathrm{h}} 10^{\mathrm{m}} 34^{\mathrm{s}} \pm 15^{\mathrm{s}}, \delta(1950)=+32^{\circ} 03^{\prime} 27^{\prime \prime} \pm 60^{\prime \prime}$. The brightest star at this position on the Palomar Observatory Sky Survey O plate has $m_{\mathrm{pg}} \sim 17$ (Katz et al. 1986).

Gamma-ray bursters are believed to flash optically (Schaefer 1985), and the possibility that the optical events reported here are the result of a gamma-ray burster cannot be excluded, but this is unlikely in view of the high repetition rate ( hours) of the optical flashes (Katz et al. 1986). A $9^{\prime} \times 9^{\prime}$ VLA "snapshot" centered on the photographic position did not show any radio source brighter than $5 \mathrm{mJy}$ (Katz et al. 1986). The area was not surveyed by the Einstein X-ray satellite (Seward and Macdonald 1983).

Two of the authors (WHGL and JvP) learned about these unusual flashes at the end of November 1985, and immediately proposed that EXOSAT (and coordinated groundbased) observations be made. We report here the results of these observations.

\section{OBSERVATIONS}

We observed the location of the photographed event with the low-energy (LE) imaging detectors (De Korte et al. $1981)$ and the medium-energy (ME) proportional-counter arrays (Turner, Smith, and Zimmermann 1983) of EXO-
$S A T$ on 5 February 1986 from UT 02:27-08:17 (ME), and UT 03:15-08:41 (LE). The LE detectors have a field of view of about $1^{\circ} \times 1^{\circ}$, and an angular resolution of $\sim 15^{\prime \prime}$; the detector was used with a Lexan-3000 filter providing an effective energy band of about $0.1-2 \mathrm{keV}$; the time resolution of our data was better than $1 \mu \mathrm{s}$. One half array $\left(\sim 750 \mathrm{~cm}^{2}\right)$ of the total ME array was pointed in a direction centered on the position of the photographed event during the first $2.6 \mathrm{hr}$ of the observations, while the other half array $\left(\sim 750 \mathrm{~cm}^{2}\right)$ was offset by $\sim 2^{\circ}$ to monitor the background counting rate. For the remaining $3.3 \mathrm{hr}$ the situation was reversed. The data were recorded in 32 energy channels covering the range 1-20 $\mathrm{keV}$; the time resolution of our ME data was $0.3 \mathrm{~s}$.

Radio observations were made by two of the authors (PAF and KFT) simultaneously with the EXOSAT observations at $22.24 \mathrm{GHz}(1.35 \mathrm{~cm})$ with the $46 \mathrm{~m}$ telescope (beamwidth 1.4 arcmin) of the Algonquin Radio Observatory* from UT 01:35-06:32. Optical observations (visually and photographically) were made under clear sky conditions by several amateur astronomers simultaneously with the EXOSAT observations (UT 00:30-06:30). Between UT 03:00 and 06:30, T. C. Weekes (private communication) made observations at the Whipple Observatory using a photometer attached to a $10 \mathrm{~m}$ reflector. The field of view was $0.5^{\circ}$ (FWHM). This equipment is capable of detecting meteors down to $m_{v}=13$ (Cook et al. 1980). The sky conditions were "poor" so that a conservative sensitivity estimate was $m_{v}=10$.

\section{DATA ANALYSIS AND RESULTS}

A cross correlation was performed of the entire image of the LE detector with its known point-spread function (De

*The Algonquin Radio Observatory is operated by the National Research Council of Canada as a national radio astronomy facility. 
Korte et al. 1981). The strongest possible "steady" point source in the error box of the photographed event (see the Introduction) could have contributed 12.35 counts in the $5.4 \mathrm{hr}$ of observing. The probability of finding such a signal (or stronger) by chance coincidence is $22 \%$. Thus, no positive detection was made. The upper limit on a possible steady $\mathrm{x}$-ray flux $(0.1-2 \mathrm{keV})$ was $2 \times 10^{-12} \mathrm{erg} \mathrm{cm}^{-2} \mathrm{~s}^{-1}$.

The x-ray light curves for both sets of data ("on-" and "off-source"), simultaneously obtained with the ME, were very similar. This indicates that the observed fluctuations (of $\sim 2$ counts $\mathrm{s}^{-1}$ on time scales of $\sim 1 \mathrm{hr}$ ) were largely (perhaps entirely) due to the background. We plotted the total counting rate in one array versus that in the other, independently for the two data sets (see above). Each set of data can be fit by a straight line, as one might expect. The difference between these lines is then a direct measure for a possible steady $x$-ray flux from a source. The $2 \sigma$ upper limit that we found in the energy range $1-20 \mathrm{keV}$ was $0.15 \mathrm{mCrab}$, corresponding to $6 \times 10^{-12} \mathrm{erg} \mathrm{cm}^{-2} \mathrm{~s}^{-1}$ (for an assumed Crab-like spectrum).

The upper limits to a possible $1 \mathrm{~s} x$-ray flash were $4 \times 10^{-9}$ (LE data; $0.1-2 \mathrm{keV}$ ), and $7 \times 10^{-10}$ (ME data; $1-20 \mathrm{keV}$ ). The upper limit ( $5 \sigma$ level of confidence) to $\mathrm{a} \geqslant 0.3 \mathrm{~s}$ radio burst was $4 \mathrm{Jy}$. No optical flashes were reported by any of the amateur astronomers (for limiting sensitivities see Katz et al. 1986), and no excess in the meteor rate was reported at the Whipple Observatory.

\section{DISCUSSION}

The 25 optical flashes reported by Katz et al. (1986) were observed in a total of $\sim 300 \mathrm{hr}$ of observing. The origin of these events (and of the photographed event) is unclear, and there is growing doubt that they are of celestial origin (Feldman, Halliday, and Blackwell 1986). Katz et al. (1986) themselves mention that it is possible that some of the flashes reported by them were not real, since psychological effects may have come into play subsequent to the visual survey (1984 October). However, they believe that this is unlikely for all reported events, particularly in view of the fact that the bright event observed visually during a photographic exposure was also recorded on film.

Vanderspek, Zachary, and Ricker (1987) made $\sim 70 \mathrm{hr}$ of observations with a CCD camera covering a $2^{\circ} \times 2^{\circ}$ field of view centered on the photographed flash; no celestial optical flashes were detected to a limiting magnitude of 7.1 for a $1 \mathrm{~s}$ flash. Anna Zytkow (private communication; manuscript in preparation) inspected $\sim 500$ Harvard archival plates covering $\sim 700 \mathrm{hr}$ (over a period of $\sim 10^{2} \mathrm{yr}$ ) of observing; no flashes were present within an area of $3^{\prime} \times 7^{\prime}$ centered on the photographed event. The limiting magnitude for an $\sim 1 \mathrm{~s}$ flash on the various plates ranges from $m \sim 2$ to $m \sim 8$. A zero- or one-magnitude flash lasting $\sim 1 \mathrm{~s}$ would not have been missed on any of the plates.

\section{CONCLUSIONS}

There is, of course, no a priori reason why the flasher, if real, would be a detectable x-ray or radio emitter. On the other hand, if an x-ray or radio source had been found at the location of the photographed event, its reality would have been beyond doubt, and we would have come closer to understanding the mystery. This, however, is not the case.

In view of the growing number of negative optical observations, it would appear to us that either the optical flashes do not have a celestial origin, or that the flasher has a transient character and turns off for extended periods (many decades) of time.

W.H.G.L. and J.vP. are grateful to Saul Rappaport for bringing the optical flashes to their attention, and to Tony Peacock for making EXOSAT time available. We thank the following people for providing ground support during the course of these observations: R. Binzel, A. Uomoto, M. Zalcik, P. Brown, B. Davis, B. Katz, A. Fullerton, E. Kindl, and D. Welch. We thank Anna Zytkow for sharing with us her findings before publication. W.H.G.L. acknowledges a generous award from the Alexander von Humboldt Stiftung, support from the United States National Aeronautics and Space Administration (grant NAG8-571), and a fellowship from the John Simon Guggenheim Memorial Foundation. J.vP. acknowledges a travel grant from the Dutch Foundation for the Advancement of Pure Science (ZWO). E.D. is financially supported by the Dutch Foundation of Astronomy (ASTRON) under contract No.782-371-019. M.L.M. is grateful to the Natural Sciences and Engineering Research Council of Canada for its continuing support.

Note added in proof: In a recent paper by P.D. Maley (Astrophys. J. Lett. (submitted)), it is shown convincingly that the light flashes seen from the Aries-Perseus region are probably all due to reflections off tumbling satellites.

\section{REFERENCES}

Cook, A. F., Weekes, T. C., Williams, J. T., and O'Mongain, E. (1980). Mon. Not. R. Astron. Soc. 193, 645.

De Korte, P. J. M., Bleeker, J. A. M., Den Boggende, A. J. F., BranduardiRaymont, G., Brinkman, A. C., Culhane, J. L., Gronenschild, E. H. M. B., Mason, I., and McKechnie, S. P. (1981). Space Sci. Rev. 30, 495.

Feldman, P. A., Halliday, I., and Blackwell, A. (1986). Private communication (manuscript in preparation).

Katz, B., Driscoll, D., Millyard, K., Waters, B., Zalcik, M., Adair, J., Gada, A., Hayes, W., Kelsch, R., McWatters, R., Rokosz, R., Zehethofer, J., Fullerton, A., Lyons, R., and McCall, M. (1986). Astrophys. J. Lett. 307, L33.
Schaefer, B. E. (1985). Astron. J. 90, 1363.

Seward, F. D., and Macdonald, A. (1983). Einstein (HEAO-2) Observing Catalog, 4th ed. (Harvard-Smithsonian Center for Astrophysics, Boston).

Turner, M. J. L., Smith, A., and Zimmermann, H. U. (1983). Space Sci. Rev. 30, 513.

Vanderspek, R. K., Zachary, D. S., and Ricker, G. R. (1987). Advances in Space Research, Proceedings of the COSPAR meeting, Toulouse, France, July 1986 (Pergamon, Oxford, NY) (in press). 